\title{
ENHANCING THE OSSEODENSIFICATION CONCEPT USING PLATELET-RICH FIBRIN IN SINUS LIFTING FOR MAXILLARY CLASS II KENNEDY CASES
}

\author{
Ahmed Mohamed El-Sayed * and Sherif Aly Sadek **
}

\begin{abstract}
Objectives: This study aimed to enhance Osseo densification by using Platelet-rich fibrin (PRF) on sinus lifting of maxillary implant in class II partially edentulous cases.

Materials and Methods: fourteen maxillary class II partially edentulous patients were selected and divided into two groups. Single implant was inserted in the edentulous region with closed sinus lifting technique for each patient in both groups, for group I implant was placed with the Densah bur drilling system together with PRF while in group II other implant was placed with that same system without PRF. Implant primary stability was measured using Resonance Frequency Analysis by Osstell immediately after torqueing the implants and every two weeks for 2 months. Rate of bone formation was followed up by taking CBCT after implant loading and followed up for one year.
\end{abstract}

Results: No significant difference was found regarding Osstell values at all follow up appointments for both groups. Regarding the bone formation, the group with PRF there was significant difference than the group without PRF.

Conclusions: Using Densah burs in closed sinus lifting consider very safely and effective technique and using PRF as grafting material in closed sinus lifting enhancing rate of bone formation.

KEYWORDS: Osseo densification, Closed sinus lifting, Platelet-rich Fibrin, Maxillary class II Kennedy

\section{INTRODUCTION}

Non extraction Osseo densification (OD) was first developed by Huwais in 2014 by using specifically designed burs to enhance bone density as they expand an osteotomy. ${ }^{(1)}$
These burs combine the merits of osteotomes with the speed and tactile control of the drilling procedures, as standard drills remove and excavate bone during implant site preparation; while osteotomes preserve bone and induce fractures of

* Associate professor of Removable Prosthodontics, Faculty of Dentistry, Cairo University.

** Lecturer of Removable Prosthodontics, Faculty of Dentistry, Cairo University. 
the trabeculae that require long remodeling time and delayed secondary implant stability. The bone preservation and condensation through compaction autografting during osteotomy preparation, usually increases the peri implant bone density $(\% \mathrm{BV})^{(2)}$

These special burs according to the manufacturer demonstrates the ability to expand narrow bone ridges similarly to split crest techniques, as the bur geometry rotating in reverse mode at a rotating speed of 800 to $1500 \mathrm{rpm}$ with profuse saline solution irrigation to prevent bone overheating. This permits the bone along the inner surface of the implant osteotomy site to be compressed without cutting. ${ }^{(3)}$

The bouncing motion (in and out movement) is helpful to create a rate-dependent stress to produce a rate dependent strain and allows saline solution pumping to gently pressurize the bone walls. This combination facilitates the enhancement of bone plasticity and expansion. ${ }^{(2)}$

Platelet rich fibrin (PRF) is one of the utmost fresh innovations in oral implantology since it is a rich source of autogenous growth factors and cytokines. PRF is mainly a concentration of platelets that are trapped in a fibrin matrix. These platelets provide a gradual and sustained release of both growth factors and cytokines. Growth factors include bone morphogenetic proteins, as well as platelet derived, vascular endothelial and transforming forms of growth factors. ${ }^{(4)}$

Such factors attract stem cells to the site of the wound promoting tissue regeneration through cell mitosis, angiogenesis and osteogenesis. This occurs in the first few weeks resulting in considerably faster healing as well as soft and hard tissue regeneration. ${ }^{(4,5)}$

Reaching enough implant initial stability in poor bone density is highly problematic. Utilizing osteotomes in deprived bone mass allows fracturing and condensing of bone trabeculae, but this technique does not improve peri implant bone density (\%BV) or implant stability. ${ }^{(6)}$

It has been documented that the fractured peri implant bone trabeculae occasioned from employing the osteotome technique, induces a delayed secondary stability with respect to conventional drilling trials throughout healing. ${ }^{(7)}$ Besides that, tooth loss, old age and removable or unsuitable removable dentures inevitably lead to alveolar bone resorption both in height and width. ${ }^{(8)}$ Furthermore, it has been reported that around 25\% of bone reduction in width take place 1-year post tooth extraction and the mandible displayed 4 times advancement in bone loss than the maxilla. ${ }^{(9)}$

Since edentulous patients characterized by owning narrow ridges demanding dental implant restoration frequently need surgical methods to perform bone expansion or augmentation. Hence, the alveolar ridge splitting/ expansion technique in 1 stage was proposed as a valid alternative to the 2-stage Guided Bone Regeneration (GBR). ${ }^{(10)}$

The predictability of both horizontal and vertical augmentation procedures by employing bone substitutes or autogenous bone is not yet clear enough and surgical difficulties are mutual. ${ }^{(11)}$ However, osteo distraction osteogenesis and ridge splitting procedure are considered efficient to enhance bone width with minimal incidence of obstacles. ${ }^{(12,13)}$

\section{MATERIALS AND METHODS}

\section{Patient Selection}

Fourteen male maxillary Kennedy class II partially edentulous patients with either canine or first premolar as last abutment and opposing dentulous mandibular arch were selected from the outpatient clinic of the Prosthodontics Department; Faculty of Oral and Dental Medicine, Cairo University.

The patients' ages ranged from 40-50 years old and were free from any systemic disease that 
may interfere or prevent implant placement and/or affects Ossteointegration.

Patients with limited bone height at least $7 \mathrm{~mm}$ below the floor of the maxillary sinus, secondary to sinus pneumatization and minimum $5 \mathrm{~mm}$ bone buccolingual width were particularly selected for prosthetic reconstruction together with sufficient inter arch space and normal maxillomandibular relation for partial denture construction. Proper selection of cooperative patients that are capable of following instructions and also with proper neuromuscular coordination were included in the study. Prior to launching the study all patients were familiarized with its nature and asked to sign consent forms.

\section{Acrylic Partial Dentures Construction}

Primary impression (Cavex Holland BV) was made for all patients then poured into stone casts. The upper study cast was preliminary surveyed to determine the path of insertion and removal, then the needed mouth preparation and special trays were constructed.

This was followed by making secondary impression (Lascod Spq, Sestofino(f1), Italy.) for the upper arch and poured into stone to get the master cast. Undesirable undercuts were blockedout using block-out wax and trimmed parallel to the path of insertion.

Jaw relation following the interocclusal wax technique was made to mount the upper and lower cast. Setting of Cross-linked acrylic teeth (Acrostone medical and dental supplies.) took place and the waxed-up denture was tried in the patient's mouth, then denture processing was carried out in the usual manner. At the delivery appointment, final occlusal adjustments and refinements were done and the denture was delivered to the patients one week before the surgical appointment to achieve sufficient patient adaptation.

\section{Implant Placement}

The finished maxillary denture can be also used as a surgical geeeeeeeeuide template to facilitate implant placement during surgery in the molar region. After flap reflection, the implant site was marked with a $2.0 \mathrm{~mm}$ pilot drill guided by the surgical guide and then prepared with a drill to a depth of $1 \mathrm{~mm}$ from the sinus floor to prevent the tip of the drill from rupturing the Schneiderian membrane. Then the sequential use of Densah Bur (Versah) (Densah bur kit.) $2.0 \mathrm{~mm}$ pilot and $2.8 \mathrm{~mm}$, multi-fluted tapered burs in a counter clockwise direction (figure 1) under copious irrigation was carried out.

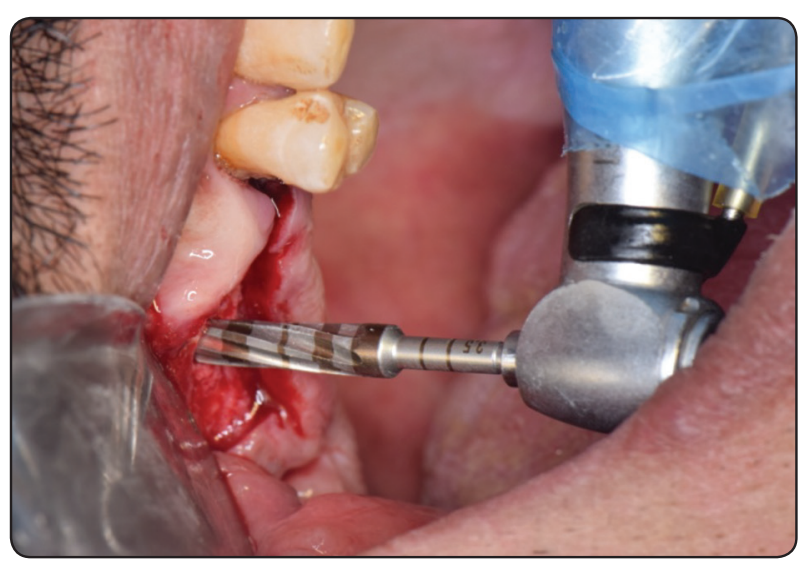

Fig. (1) Multi-fluted Densah tapered burs in a counter clockwise direction.

As the next wider densah burs (3.5mm) advancement in the osteotomy was carried out. Bone was pushed towards the apical end and began to gently lift the membrane and autograft compacted bone to achieve additional vertical depth and membrane lift of at least $2.0 \mathrm{~mm}$ and reached the final desired width for implant placement.

Preparation of the PRF grafting material for the second implant was then commenced as described by Choukroun ${ }^{(14)}$. A $10 \mathrm{~mm}$ blood sample was drawn from each patient into a sterile red plain (without anticoagulant) test tube. The test tube was then centrifuged at $3000 \mathrm{rpm}$ for 12 minutes (Process, 
Nice, France). The PRF clot was handled using pliers and separated from the red blood cell clot. It was then placed on the metal grid in the PRF Box (Process Ltd., Nice, France). Using the condenser apparatus, the clot was compressed to produce the PRF membrane.

\section{The patients were divided into 2 groups:}

The fourteen patients were divided into two groups, single implant was inserted in the edentulous region with closed sinus lifting technique for each patient; in both groups, for group I implant was placed with the Densah bur drilling system together with PRF(figure 2) while in group II other implant was placed with that same system without PRF.

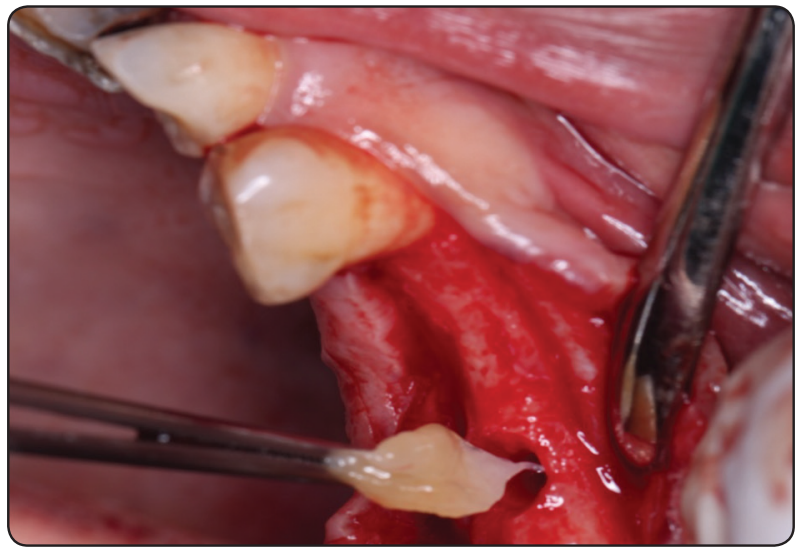

Fig. (2) PRF fragment before implant placement.

The implant of $3.6 \mathrm{~mm}$ in diameter, $10 \mathrm{~mm}$ in length were placed using the torque wrench in each osteotomy site. (Dentium implant system (Dentium, Samsung-dong, Seoul, Korea)). (Figure 3).

Attachment of the smart peg on the implants and the Osstell was used to record the initial stability ISQ and between (0w) and each of (2w), (4w), (6w) and $(8 w)$ groups. Figure (4)

Suturing took place around the healing collars. All patients received $2 \mathrm{gm} /$ day amoxicillin clavulanate and $50 \mathrm{mg} / 8$ hours non-steroidal antiinflammatory analgesics for 5 days postoperatively. Postoperative instructions were given including

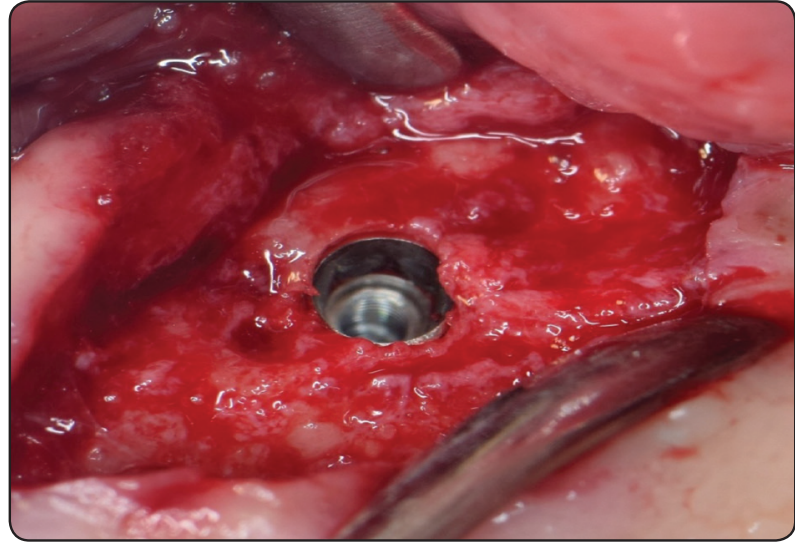

Fig. (3) The implant of $3.6 \mathrm{~mm}$ in diameter, $10 \mathrm{~mm}$ in length were placed using the torque wrench in each osteotomy site.

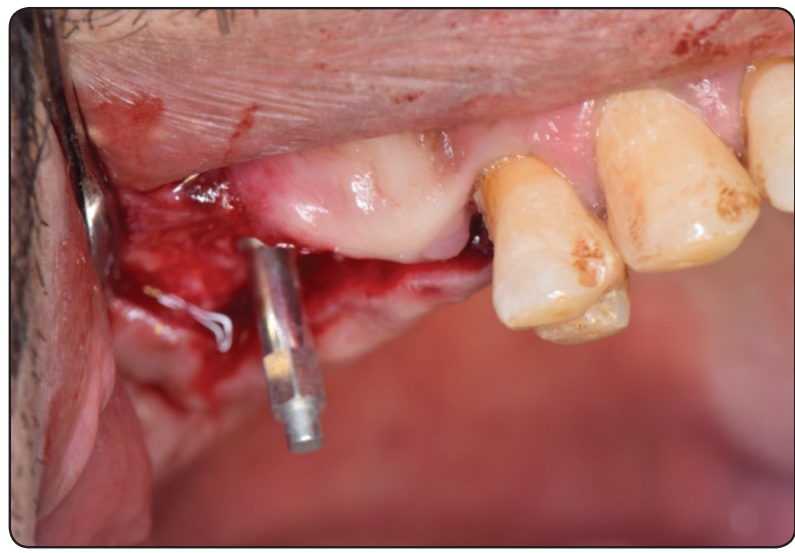

Fig. (4) Attachment of the smart peg on the implants and the Osstell was used to record the initial stability ISQ,

a soft diet and appropriate oral hygienic measures with $0.2 \%$ chlorhexidine mouth rinse.

\section{Overdenture Pick-up}

The denture was checked to fit over the implants with their housings without interfering with its original fit. A small hole was done in its palatal surface near to site of implant to allow escape of excess acrylic material during refitting. The healing collar were refitting into the mucosal surfaces of the overdenture using a Hard-Pick-up material (Secure HARD reline kit 3MTMESPETM, Germany). The patients were instructed to occlude on the dentures till the material set. Any excess material was removed and the denture was finished and polished. 
Patients in all groups received CBCT immediately after the implant loading and followed up for one year at 6 months and 12 months intervals. Each implant was evaluated for the rate of bone formation around the implant, peri-implant bone quality and bone surrounding implant apices. The radiographs were compared with the base line ones taken immediately after surgery and followed up for one year at 6 months and 12 months intervals.

\section{RESULTS}

The mean and standard deviation values were calculated for each group in each test. Data were explored for normality using Kolmogorov-Smirnov and Shapiro-Wilk tests, data showed parametric (normal) distribution.

Independent sample t-test was used to compare between two groups in non-related samples. Repeated measure ANOVA was used to compare between more than two groups in related samples. Paired sample t-test was used to compare between two groups in related samples.

Two-way ANOVA was used to test the interaction between variables.

The significance level was set at $\mathrm{P} \leq 0.05$. Statistical analysis was performed with IBM $^{\circledR}$

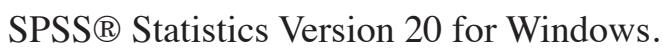

\section{Stability results:}

\section{A) Effect of time:}

\section{Group I (With PRF):}

There was no statistically significant difference between $(0 w),(2 w),(4 w),(6 w)$ and $(8 w)$ groups where $(p=0.081)$.

\section{Group II (Without PRF):}

There was no statistically significant difference between $(0 \mathrm{w}),(2 \mathrm{w}),(4 \mathrm{w}),(6 \mathrm{w})$ and $(8 \mathrm{w})$ groups where $(p=0.082)$.

\section{B) Effect of groups:}

\section{0w:}

There was no statistically significant difference between (Group I) and (Group II) groups where $(p=0.296)$.

\section{2w:}

There was no statistically significant difference between (Group I) and (Group II) groups where $(p=0.548)$.

\section{4w:}

There was no statistically significant difference between (Group I) and (Group II) groups where $(p=0.696)$.

\section{6w:}

There was no statistically significant difference between (Group I) and (Group II) groups where $(p=0.223)$.

\section{8w:}

There was no statistically significant difference between (Group I) and (Group II) groups where $(p=0.509)$.

TABLE (1): The mean, standard deviation (SD) values of stability of different groups.

\begin{tabular}{|l|c|c|c|c|c|}
\hline \multirow{4}{*}{ Variables } & \multicolumn{4}{|c|}{ Stability } \\
\cline { 2 - 5 } & \multicolumn{2}{|c|}{$\begin{array}{c}\text { Group I } \\
\text { With PRF }\end{array}$} & \multicolumn{2}{|c|}{$\begin{array}{c}\text { Group II } \\
\text { Without PRF }\end{array}$} & \multirow{2}{*}{ p-value } \\
\cline { 2 - 5 } & Mean & SD & Mean & SD & \\
\hline \multirow{2}{*}{$\mathbf{w}$} & 67.90 & 1.91 & 67.10 & 1.37 & $\mathbf{0 . 2 9 6 n s}$ \\
\hline $\mathbf{2 w}$ & 66.50 & 5.10 & 65.20 & 4.37 & $\mathbf{0 . 5 4 8 n s}$ \\
\hline $\mathbf{4 w}$ & 65.00 & 3.30 & 64.50 & 2.22 & $\mathbf{0 . 6 9 6 n s}$ \\
\hline $\mathbf{6 w}$ & 64.70 & 2.00 & 63.60 & 1.90 & $\mathbf{0 . 2 2 3 n s}$ \\
\hline $\mathbf{8 w}$ & 63.30 & 2.75 & 62.50 & 2.55 & $\mathbf{0 . 5 0 9 n s}$ \\
\hline $\boldsymbol{p}$-value & $\mathbf{0 . 0 8 1 n s}$ & \multicolumn{5}{|c|}{$\mathbf{0 . 0 8 2 n s}$} & \\
\hline
\end{tabular}

*; significant $(p<0.05) \quad n s ;$ non-significant $(p>0.05)$ 


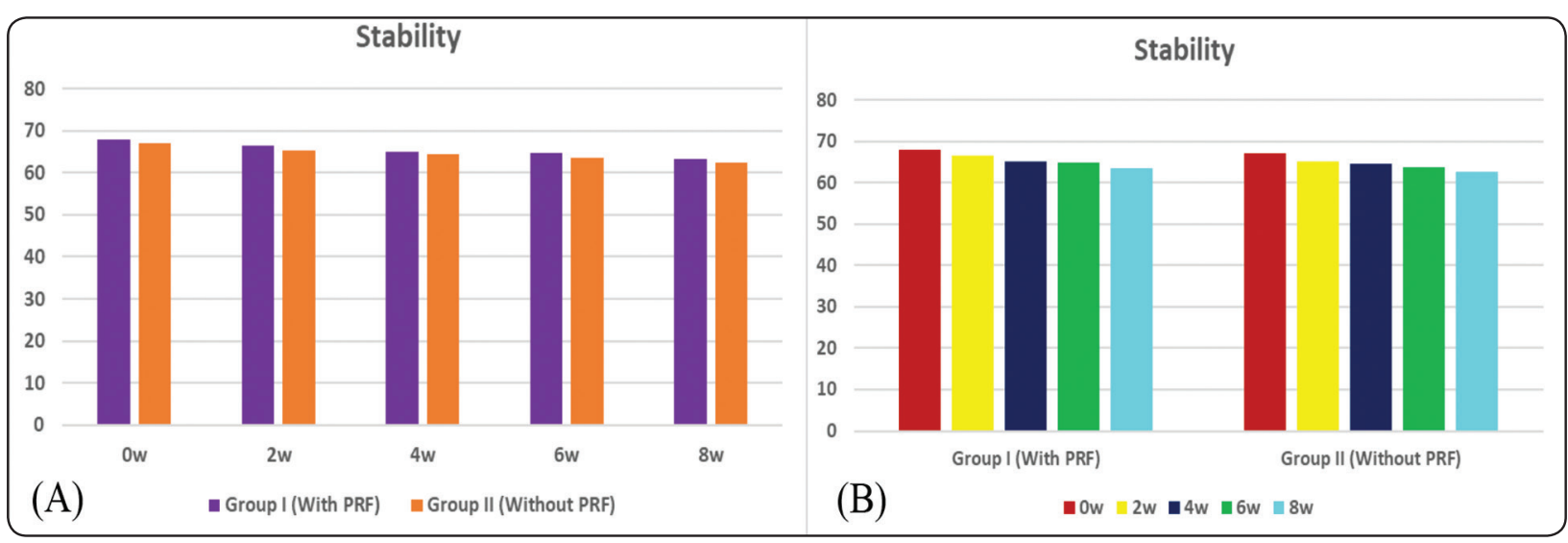

Fig. (5): Bar chart representing stability for different groups

TABLE (2): Results of Two-way ANOVA for the effect of different variables on mean Stability.

\begin{tabular}{|c|c|c|c|c|c|}
\hline Source of variation & $\begin{array}{c}\text { Type III } \\
\text { sum of Squares }\end{array}$ & df & $\begin{array}{c}\text { Mean } \\
\text { Square }\end{array}$ & $F$ - value & $P$-value \\
\hline Groups & 20.250 & 1 & 20.250 & 2.297 & .133 \\
\hline Time & 243.260 & 4 & 60.815 & 6.898 & .100 \\
\hline Groups x Time & 1.900 & 4 & .475 & .054 & .994 \\
\hline
\end{tabular}

$d f:$ degrees of freedom $=(n-1), *$ Significant at $P \leq 0.05$

\section{Two-way ANOVA:}

Data in table (2) shows the results of Two-way ANOVA analysis for the effect of different variables on stability. The results showed that groups had no statistically significant effect. Also, time had no statistically significant effect. The interaction between the two variables had no statistically significant effect.

\section{Bone formation results:}

\section{A) Effect of time:}

\section{Group I (With PRF):}

There was a statistically significant difference between $(0 \mathrm{~m}),(6 \mathrm{~m})$ and $(12 \mathrm{~m})$ groups where $(p<0.001)$.

A statistically significant difference was found between $(0 \mathrm{~m})$ and each of $(6 \mathrm{~m})$ and $(12 \mathrm{~m})$ groups where $(\mathrm{p}<0.001)$.
Also, a statistically significant difference was found between $(6 \mathrm{~m})$ and $(12 \mathrm{~m})$ groups where $(p=0.001)$.

\section{Group II (Without PRF):}

There was a statistically significant difference between $(0 \mathrm{~m}),(6 \mathrm{~m})$ and $(12 \mathrm{~m})$ groups where $(p<0.001)$.

A statistically significant difference was found between $(0 \mathrm{~m})$ and each of $(6 \mathrm{~m})$ and $(12 \mathrm{~m})$ groups where $(\mathrm{p}=0.011)$ and $(\mathrm{p}<0.001)$

Also, a statistically significant difference was found between $(6 \mathrm{~m})$ and $(12 \mathrm{~m})$ groups where $(p=0.002)$.

\section{B) Effect of groups:}

\section{0m:}

There was no statistically significant difference between (Group I) and (Group II) groups where $(p=0.823)$. 


\section{6m:}

There was a statistically significant difference between (Group I) and (Group II) groups where $(p=0.042)$.

\section{12m:}

There was a statistically significant difference between (Group I) and (Group II) groups where $(p=0.009)$.

\section{Two-way ANOVA:}

Data in table (4) shows the results of Two-way ANOVA analysis for the effect of different variables on stability. The results showed that groups had a statistically significant effect. Also, time had a statistically significant effect. The interaction between the two variables had no statistically significant effect.

TABLE (3): The mean, standard deviation (SD) values of bone formation of different groups.

\begin{tabular}{|c|c|c|c|c|c|}
\hline \multirow{3}{*}{ Variables } & \multicolumn{5}{|c|}{ Bone formation } \\
\hline & \multicolumn{2}{|c|}{ Group I With PRF } & \multicolumn{2}{|c|}{ Group II Without PRF } & \multirow[t]{2}{*}{ p-value } \\
\hline & Mean & SD & Mean & SD & \\
\hline $\mathbf{0 m}$ & 1.55 & 0.32 & 1.51 & 0.23 & $0.823 n s$ \\
\hline $6 \mathrm{~m}$ & 2.31 & 0.24 & 1.93 & 0.26 & $0.042 *$ \\
\hline $12 \mathrm{~m}$ & 2.74 & 0.17 & 2.40 & 0.14 & $0.009 *$ \\
\hline$p$-value & \multicolumn{2}{|c|}{$<0.001 *$} & \multicolumn{2}{|c|}{$<0.001 *$} & \\
\hline
\end{tabular}

*; significant $(p<0.05) \quad n s ;$ non-significant $(p>0.05)$

TABLE (4): Results of Two-way ANOVA for the effect of different variables on mean Bone formation.

\begin{tabular}{|c|c|c|c|c|c|}
\hline Source of variation & $\begin{array}{c}\text { Type III } \\
\text { sum of Squares }\end{array}$ & df & $\begin{array}{c}\text { Mean } \\
\text { Square }\end{array}$ & $F$ - value & $P$ - value \\
\hline Groups & .479 & 1 & .479 & 8.889 & .006 \\
\hline Time & 5.415 & 2 & 2.707 & 50.260 & .000 \\
\hline Groups x Time & .172 & 2 & .086 & 1.599 & .223 \\
\hline
\end{tabular}

$d f:$ degrees of freedom $=(n-1), *$ Significant at $P \leq 0.05$

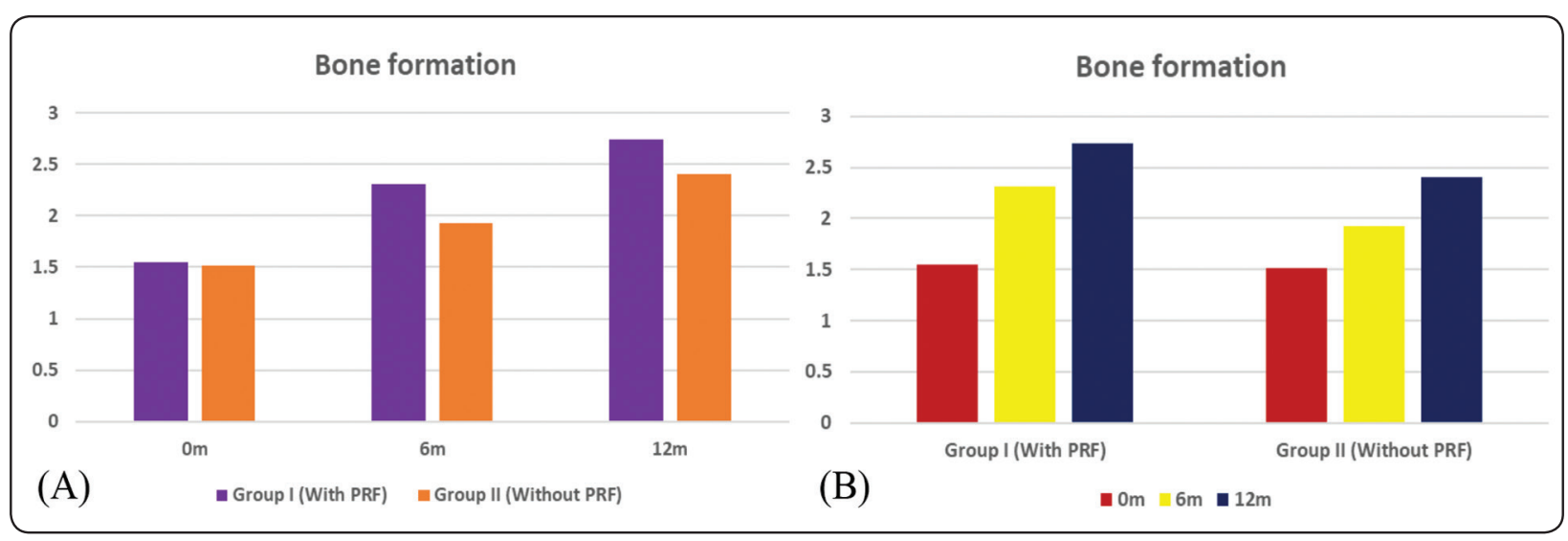

Fig. (6): Bar chart representing bone formation for different groups 


\section{DISCUSSION}

The selected patients were apparently healthy with proper general appraisal and no medical history of debilitating diseases to avoid the adverse effect of systemic disorders on both the healing process and osseointegration.

In this study RFA (Resonance Frequency Analysis) method was utilized for monitoring implant stability on the surgical daytime by aid of implant stability quotient (ISQ) value reading, through Osstell device.

Closed sinus lift technique was recommended than the open one due to its minimal invasiveness that enables placement of implants of $10 \mathrm{~mm}$ in length or longer simultaneously with reduction on osteotomy. This provides better postoperative comfort and preservation of the sinus cavity integrity compared with the open one. ${ }^{(14)}$

Selected cases had at least $7 \mathrm{~mm}$ of remaining alveolar bone height between the antrum's floor and alveolar crest as bone length is a chief reason for proper engagement and initial stabilization of implant in closed sinus lift technique with simultaneous implant. ${ }^{(15)}$

In the current study, we elevated the sinus membrane 2 to $3 \mathrm{~mm}$ free from any graft supplementation in group 1 to avoid the risk of tearing or puncturing the membrane and with addition of PRF in group 2. This promotes new bone formation on implant's apical part through the osteogenic potentiality of the Schneiderian membrane that is self-possessed of limited layers including the epithelial lining, lamina propria and osteogenic layer towards the maxillary bone. ${ }^{(16,17)}$

According to Lai et al.,2010 implant's protrusion into the sinus deprived of any grafting materials plays a major role in the amount of bone gain and complete regeneration takes place over the entire surface when implants protruded $2-3 \mathrm{~mm}$ into the sinus. ${ }^{(18)}$
Osseo densification has the ability of raising the sinus membrane with minimal perforation hazard and simplifying the autogenous bone grafting to enhance implant stability. These capabilities are based on a combination of the following technical processes.

Hydrodynamic wave action from fluid pumping coupled with high-speed counterclockwise drill rotation induce effluence ahead of the point of contact. Once the sinus floor is penetrated by the densifying bur irrigation solution and bone debris serve to hydraulically elevate the sinus membrane. ${ }^{(19)}$

The consequences of this consideration reveal that group 2 that contains PRF material have an encouraging impact on both implant stability and rate of bone development. These results are supported by the strong evidence in the literature regarding the effect of PRF on wound healing and tissue regeneration, especially in the early healing phase. ${ }^{(20)}$

The present study also reaches an agreement with the finding of Shaarawy and Fahmy ${ }^{(21)}$ that displayed a remarkable growth in peri-implant bone density post PRF grafting of immediately placed implants when compared to synthetic bone substitutes. They also agree with those by Oncu et al ${ }^{(20)}$ which demonstrated that PRF enlarged both amount and rate of new bone creation and enhanced bone to implant contact throughout the initial stages of healing.

\section{CONCLUSIONS}

\section{Within limitation of this study}

1- Using Densah burs in closed sinus lifting very safely and effective technique.

2- Using PRF as grating material in closed sinus lifting enhancing rate of bone formation.

\section{RECOMMENDATION}

Further study and more follow up periods are recommended. 


\section{REFERENCES}

1. Huwais S. Autografting Osteotome. Geneva, Switzerland: World Intellectual Property Organization Publication; 2014.WO2014/077920.

2. Huwais S, Meyer E. Osseodensification: A novel approach in implant o preparation to increase primary stability, bone mineral density and bone to implant contact. Int J Oral Maxillofac Implants. 2015.

3. Tian JH, Neiva R, Coelho PG, Witek L, Tovar NM, Lo IC, Gil LF and Torroni A. Alveolar Ridge Expansion: Comparison of Osseodensification and Conventional Osteotome Techniques. J Craniofac Surg. 2019;30(2):607-610.

4. Antonio Cortese, Giuseppe Pantaleo, Massimo Amato, Candace M. Howard, Lorenzo Pedicini and Pier Paolo Claudio. Platelet-rich fibrin (PRF) in implants dentistry in combination with new bone regenerative flapless technique: evolution of the technique and final results. Open Med. 2017; 12: 24-32

5. Simonpieri A, Del Corso M, Vervelle A, Jimbo R, Inchingolo F, Sammartino G and Dohan Ehrenfest DM. Current knowledge and perspectives for the use of plateletrich plasma (PRP) and platelet-rich fibrin (PRF) in oral and maxillofacial surgery part 2: Bone graft, implant and reconstructive surgery. Curr Pharm Biotechnol. 2012; 13(7):1231-56.

6. Shalabi MM, Wolke JG and Ruijter AJ. Histological evaluation of oral implants inserted with different surgical techniques into trabecular bone of goats. Clin Oral Implants Res. 2007;18: 489-495.

7. Büchter A, Kleinheinz J and Wiesmann HP. Biological and biomechanical evaluation of bone remodelling and implant stability after using an osteotome technique. Clin Oral Implants Res. 2005; 16:1-8.

8. Hansson $\mathrm{S}$ and Halldin A. Alveolar ridge resorption after tooth extraction: A consequence of a fundamental principle of bone physiology. J Dent Biomech. 2012; 3 : 1758736012456543 .

9. Carlsson G and Persson G. Morphologic changes of the mandible after extraction and wearing dentures: A longitudinal clinical and $\mathrm{x}$-ray cephalometric study covering 5 years. Odontol Revy. 1967; 18:27-54.

10. Bassetti MA, Bassetti RG and Bosshardt DD. The alveolar ridge splitting/ expansion technique: A systematic review. Clin Oral Implants Res. 2015 Jan 14. [Euphaeid of print].

11. Esposito M, Grusovin MG and Felice P. The efficacy of horizontal and vertical bone augmentation procedures for dental implants: A Cochrane systematic review. Eur J Oral Implantol. 2009; 2:167-184.

12. Stricker A, Fleiner J and Stübinger S. Bone loss after ridge expansion with or without reflection of the periosteum. Clin Oral Implants Res. 2015; 26:529-536.

13. Tang YL, Yuan J and Song YL. Ridge expansion alone or in combination with guided bone regeneration to facilitate implant placement in narrow alveolar ridges: A retrospective study. Clin Oral Implants Res. 2015; 26:204-211.

14. Dohan DM, Choukroun J and Diss A. Platelet-rich fibrin (PRF): A second-generation platelet concentrate. Part I: Technological concepts and evolution. Oral Surgery, Oral Med Oral Pathol Oral Radiol Endodontology; 101. Epub ahead of print 2006. DOI: 10.1016/j.tripleo.2005.07.008.

15. Davarpanah, Mithridade, Henry Martinez, Jean-François Tecucianu, Georges Hage, and Richard Lazzara. "The modified osteotome technique." International Journal of Periodontics \& Restorative Dentistry 21, no. 6 (2001).

16. Srouji, S., T. Kizhner, D. Ben David, M. Riminucci, P. Bianco, and E. Livne. "The Schneiderian membrane contains osteoprogenitor cells: in vivo and in vitro study." Calcified tissue international 84, no. 2 (2009): 138-145.

17. Si, Mi-si, Long-fei Zhuang, Ying-Xin Gu, Jia-ji Mo, Shichong Qiao, and Hong-Chang Lai. "Osteotome sinus floor elevation with or without grafting: a 3-year randomized controlled clinical trial." Journal of clinical periodontology 40, no. 4 (2013): 396-403.

18. Lai, Hong Chang, Long-Fei Zhuang, Xiao-Fei Lv, ZhiYong Zhang, Yun-Xin Zhang, and Zhi-Yuan Zhang. "Osteotome sinus floor elevation with or without grafting: a preliminary clinical trial." Clinical Oral Implants Research 21, no. 5 (2010): 520-526.

19. Huwais, Salah, Ziv Mazor, Andreas L. Ioannou, Howard Gluckman, and Rodrigo Neiva. "A Multicenter Retrospective Clinical Study with Up-to-5-Year Follow-up Utilizing a Method that Enhances Bone Density and Allows for Transcrestal Sinus Augmentation Through Compaction Grafting." International Journal of Oral \& Maxillofacial Implants 33, no. 6 (2018).

20. Öncü E, Bayram B and Kantarci A. Positive effect of platelet rich fibrin on osseointegration. Med Oral Patol Oral Cir Bucal 2016; 21: e601-7.

21. Mohammed A. Shaarawy and Ahmed Fahmy. Evaluation of two different grafting materials used during immediate post-extraction implant placement for retaining mandibular overdentures. EDJ 2013; 59: 1631. 